\title{
EL PANORAMA DEL TRABAJO DECENTE EN EL PERÚ DURANTE EL PERIODO 2010 AL 2016
}

\author{
THE LANDSCAPE OF DECENT WORK IN PERU DURING THE PERIOD \\ 2010 TO 2016
}

(D) Uriel Rigoberto Quispe Quezada ${ }^{*}$, (DVladimir Rafael Villarroel Díaz ${ }^{2}$

\author{
uquispe@unah.edu.pe, vladynan@gmail.com \\ ${ }^{1}$ Universidad Nacional Autónoma de Huanta, Ayacucho, Perú \\ ${ }^{2}$ Ministerio de Transportes y Comunicaciones, Lima, Perú
}

*Correspondencia: Uriel Rigoberto Quispe Quezada. Email: uquispe@unah.edu.pe

Recibido: 01.12.19| Aprobado: 04.12.19

\section{RESUMEN}

En este artículo se ha llevado a cabo una revisión descriptiva sobre el trabajo decente en el Perú con la intención de formular un panorama general respecto al estado situacional de este tipo de empleo y establecer qué aspectos están limitando su avance y desarrollo en el país. La investigación se ha desarrollado mediante la búsqueda bibliográfica de artículos académicos, informes de autoridades competentes en la materia y al análisis de las estadísticas de algunos indicadores socioeconómicos relacionados con el trabajo decente principalmente del periodo 2010 al 2016. Los resultados obtenidos fruto de la investigación indican que existe un déficit significativo de trabajo decente tanto a nivel nacional y regional que afecta a un porcentaje importante de la población ocupada en el Perú debido principalmente a los altos niveles de informalidad y de pobreza presentes en nuestra economía. Esto evidencia que existe la urgente necesidad de orientar las políticas de Estado al mejoramiento de las condiciones laborales y la educación de la población en el país.

Palabras clave: 'Trabajo decente, déficit, informalidad, pobreza, Pe.

\begin{abstract}
In this article, a descriptive review on decent work in Peru has been carried out with the intention of formulating an overview of the situational status of this type of employment and establishing what aspects are limiting its progress and development in the country. The research has been developed through the bibliographic search of academic articles, reports of competent authorities on the subject and the analysis of the statistics of some socioeconomic indicators related to decent work mainly from the period 2010 to 2016. The results obtained as a result of the research indicate that there is a significant deficit of decent work at national and regional level that affects an important percentage of the employed population in Peru because of the high levels of informality and poverty present in our economy. This shows that there is an urgent need to orient State policies to improve working conditions and education of the population in the country.
\end{abstract}

Keywords: Decent work, deficit, informality, poverty, Peru. 


\section{INTRODUCCIÓN}

La Agenda 2030 puede ser descrita como una agenda para la sostenibilidad del desarrollo que tiene como prioridad las personas y el planeta, esta agenda fue adoptada en el año 2015 por las Naciones Unidas con la finalidad de abordar los grandes desafíos que enfrenta la humanidad incluyendo los relacionados con la problemática del mundo del trabajo considerando que se deben de crear cerca de 600 millones de nuevos empleos hacia el 2030 solamente para igualar el ritmo de crecimiento de la población en edad de trabajar, en tal sentido, la importancia del trabajo decente se ha puesto de manifestó de esta agenda con la finalidad de promover el crecimiento económico sostenible e inclusivo y el trabajo decente para todos (OIT, 2018). De acuerdo con ello, el trabajo decente puede definirse como aquel trabajo que es digno y es productivo en condiciones de libertad, equidad, seguridad y dignidad, en la cual los derechos de los trabajadores son protegidos y que cuenta con una remuneración adecuada y protección social; esta nueva visión del trabajo busca que se forme una conciencia sobre la importancia de que las personas tengan un trabajo y que éste sea decente, es decir, es una concepción del trabajo que pretende tener un alcance universal que reconoce los derechos de las personas y se dirige a todo tipo de trabajadores (Gálvez Santillán, Gutiérrez Garza, Picazzo Palencia, \& Osorio Calderón, 2016).

A pesar de ello, la problemática social en el mundo entero se agudiza año tras año, el desempleo mundial es significativamente alto y alcanza a 200 millones de personas, mientras que cientos de millones más son trabajadores pobres, asimismo, los cambios tecnológicos vienen transformando los métodos estándar del trabajo, entre otros problemas que vienen afectando la realización del trabajo decente a nivel mundial, pero para hacer realidad la visión del desarrollo sostenible en las sociedades se debe promover también el trabajo decente porque con empleos decentes se incrementará el poder adquisitivo de las personas y de las familias y con este poder adquisitivo se incentiva el crecimiento de las empresas, y a su vez, aumentarán los ingresos fiscales para los gobiernos que pueden financiar medidas sociales dirigidas a proteger a quienes no encuentren empleo, por tanto, el trabajo decente es un motor para el desarrollo sostenible de las sociedades (OIT, 2017).

En el caso peruano, independientemente del enfoque metodológico utilizado para evaluar los avances del trabajo decente, se han realizado mediciones del trabajo decente a nivel nacional y regional como las investigaciones presentadas por Gamero (2014) y Goyzueta et al (2016), no obstante a todas estas evaluaciones, se requiere complementariamente explorar estadísticas de diferentes indicadores socioeconómicos relacionados con los componentes del trabajo decente en el país como: el contexto económico, las oportunidades de empleo, los niveles de remuneraciones de los trabajadores, la seguridad social y los niveles de dialogo social para lograr una mejor comprensión del estado del trabajo decente en el Perú, así como también, nos ayudará a determinar qué aspectos vienen limitando sus avances en nuestra economía.

En consecuencia, el objetivo del presente artículo es efectuar una revisión descriptiva sobre el trabajo decente en el Perú que nos permita construir un panorama general del estado actual de este tipo de empleo en nuestro país tanto a nivel nacional como a nivel regional mediante la búsqueda bibliográfica de artículos académicos e 
informes de autoridades competentes relacionados con el trabajo decente, y la exploración y análisis de estadísticas de algunos indicadores socioeconómicos relacionados con los componentes del trabajo decente en el país principalmente del periodo 2010 al 2016, para lo cual consideramos de forma consecutiva los siguientes temas: la conceptualización e interpretación del trabajo decente, el trabajo decente como una aspiración y meta global, la problemática que enfrenta el trabajo decente, y el panorama y medición del trabajo decente en el Perú. Finalmente se esbozan algunas conclusiones respecto a la temática tratada en este artículo.

\section{TRABAJO DECENTE: CONCEPTOS E INTERPRETACIONES}

El término "trabajo decente" fue dado a conocer por primera vez en la Memoria del Director General -de la Organización Internacional del Trabajo- a la 87ª Reunión de la Conferencia Internacional del Trabajo celebrada en el año 1999; este término engloba cuatro elementos: el empleo, la protección social, los derechos de los trabajadores y el dialogo social, es decir, este concepto abarca todo tipo de trabajo (asalariado, informal, independiente, etc.), que existen empleos suficientes (posibilidades de trabajar), que es remunerado (en metálico y en especie), que los trabajadores tienen seguridad social, que se toma en cuenta sus derechos fundamentales, y la oportunidad de expresarse sobre todo asunto relacionado con la actividad laboral (Ghai, 2003).

La noción formulada por el Director General de la OIT de esa época puede calificarse como la primera utilización formal del referido término, en todo caso, el concepto de trabajo decente se relaciona con la cantidad y calidad suficiente de trabajo, que es apropiado, digno, justo, que incluye el respeto de los derechos, ingresos y condiciones satisfactorias de trabajo, protección social y un contexto de libertad sindical y de dialogo social. Asimismo, la formación es un requisito fundamental para el acceso al empleo de calidad, por tanto, parte esencial del trabajo decente, ya que no habrá trabajo decente sin la formación adecuada, por ende, tampoco será posible alcanzar altos niveles de productividad y competitividad sin inversión en capacitación, y sin o hay trabajo suficiente y decente en todo el mundo, los países desarrollados verán crecer indefinidamente sus problemas de inmigración no deseada, en todo caso, es innegable que existe una relación entre educación y trabajo de decente (Ermida Uriarte, 2001).

En todo caso, la noción de trabajo decente es un término que aún puede llenarse de contenido o que puede integrar conceptos previos antes enunciados, es decir, este término puede englobar una visión integral, ética, universal y dinámica. Sin embargo, el trabajo decente ha sido utilizado como sinónimo de trabajo digno o trabajo genuino, ambas adjetivaciones se utilizan indistintamente pero que guardan relación con las preferencias de quiénes y dónde se aplican, en el caso del concepto trabajo digno se usa mayormente en discursos sindicales que reflejan las preocupaciones relacionadas con el resguardo al trabajador y su familia, en cuanto al trabajo genuino, su utilización es de manera reivindicativa entre los excluidos y su derecho de tener trabajo. Esto indicaría que esta discusión debe centrarse más en su consistencia que en la connotación del término, es una tarea que debe incluir los distintos componentes del trabajo, y la participación de los trabajadores, empleadores y el propio Estado que es de necesidad para definir un eje en el desarrollo de este concepto (Lanari, 2005). 
Según Gálvez Santillán et al (2011), existen dos cualidades en el concepto de trabajo decente, en primer lugar, representa un piso mínimo en cuanto a la calidad del empleo que todas las personas deberían disfrutar para poder desplegar las capacidades que requieran satisfacer, sus necesidades, elevar su productividad y contribuir a fortalecer la cohesión social; y en segundo lugar, representa una aspiración que las sociedades, de forma tal que, puedan utilizarla como un medio para orientar las políticas laborales que eleven las capacidades de los trabajadores para avanzar hacia una sociedad justa y una economía productiva.

En tal sentido, no sólo debe importar el número de empleos que se crean, sino también la calidad de los mismos, las políticas públicas sin el enfoque adecuado, pueden trastocar la realización de las capacidades de la población, pueden generar exclusiones, restringen el mercado, debilitan la cohesión social y merman las instituciones. Por tanto, pensar en la oferta de trabajo bajo un enfoque de trabajo decente puede ser una estrategia relevante hacia la transición de sociedades que buscan disminuir la desigualdad social (Gálvez Santillán, Gutiérrez Garza, \& Picazzo, El trabajo decente: nuevo paradigma para el fortalecimiento de los derechos sociales, 2011).

\section{EL TRABAJO DECENTE ES UNA ASPIRACIÓN Y UNA META GLOBAL}

El trabajo es un aspecto trascendental para las personas -según Ballestero (2016) por eso que es necesario establecer condiciones mínimas que dignifiquen el empleo, en tal sentido, el trabajo decente establece una línea por debajo de la cual no sería licito desempeñar ningún trabajo, lo que indica que todos los trabajadores independientemente de dónde o qué tipo de trabajo realicen tienen derechos laborales que deben ser garantizados y respetados, esto refleja la patente de universalidad que tiene el concepto de trabajo decente, por tanto, apostar por él, no sólo traería beneficios individuales para cada trabajador, sino también para todo el resto de la sociedad, puesto que el trabajo decente resultará en un aumento de los salarios de las familias, ingresos que finalmente repercuten en el crecimiento económico, en definitiva, el trabajo decente deberá ser una aspiración del siglo XXI si se desea lograr un desarrollo económico sostenible.

\section{PROBLEMAS, POLÍTICAS Y DÉFICIT DEL TRABAJO DECENTE}

Según Egger et al (2002), el trabajo decente puede considerase como una meta que se han impuesto varias sociedades, y a la vez puede ser un marco para crear las políticas necesarias que conduzcan al objetivo de trabajo decente para las personas, las políticas de trabajo decente deben estar formuladas en base a una serie de instrumentos internacionales tales como la Declaración Universal de los Derechos Humanos, el Pacto Internacional sobre Derechos Económicos, Sociales y Culturales, la Declaración de la Organización Internacional del Trabajo sobre Principios y Derechos al Trabajo, .entre otros, es decir, crear políticas tendientes a convertir las aspiraciones por tener un trabajo decente en la realidad; sin embargo, muchas veces, el trabajo es tratado como una simple mercancía que forma parte de un mercado, pero el trabajo justo es una dimensión importante de la estabilidad social y la paz, para la productividad y la competitividad que son factores determinantes para un crecimiento económico sostenido; la problemática del mundo actual afecta la realización del trabajo decente; como cuando existe limitaciones en el acceso al empleo se fomenta la sumisión, la marginalización y la pobreza; cuando 
existe discriminación por sexo, nacionalidad, raza o edad; hostigamiento sexual; cuando la remuneración no es suficiente para subsistir o existe desigualdad de ingresos; cuando existe sobrexplotación del trabajador; cuando no existe un ambiente adecuado para trabajar.

El déficit de trabajo decente existe cuando hay: una brecha del empleo que se caracteriza por el desempleo y el subempleo pero a gran escala; una brecha de los derechos cuando existe una denegación generalizada de los derechos en el trabajo; una brecha de la protección social cuando existen condiciones de trabajo inseguras y también en la inseguridad de los ingresos; y una brecha del dialogo social cuando existe una representación inadecuada y falta de instituciones en las que la gente pueda hacer oír su voz; si no se reduce el déficit, la meta de justicia social que se desea lograr estará fuera del alcance (Somavía, 2001).

\section{EL PANORAMA Y MEDICIÓN DEL TRABAJO DECENTE EN EL PERÚ}

A fin de formarnos un panorama del trabajo decente en el Perú, utilizaremos estadísticas de algunos indicadores socioeconómicos del Perú relacionados con el concepto de trabajo decente: el contexto económico, oportunidades de empleo; niveles de remuneraciones; seguridad social; y libertad sindical y negociaciones colectivas en el Perú.

En primer lugar, respecto al contexto económico en el Perú; en el año 2016, se observó un incremento en la tasa de crecimiento del PBI de 3.91\%, esta tendencia creciente se inició en el año 2014, aunque es una tasa menor respecto a las tasas de históricas de mayor crecimiento registradas en los años 2008 y 2010 con $9.14 \%$ y $8.45 \%$ respectivamente. Ver la Figura 01.

\section{Figura 01}

Variación porcentual del PBI del Perú: 2005 - 2016.



Fuente: BRCP. Tomado de Plades (2016).

En segundo lugar, respecto a las oportunidades de empleo en el Perú. En la Figura 02 se observa la evolución de la pobreza y pobreza extrema en el Perú -entendida como la insuficiencia de recursos monetarios para adquirir una canasta de bienes y servicios aceptable-, en el año 2016, la pobreza a nivel nacional disminuyó en 1\%, sin embargo, el 
$20.7 \%$ de la población se encuentra en situación de pobreza. Respecto a la pobreza extrema, se observa que el 4.8\% de la población peruana es pobre extremo (2016), los pobres extremos en el Perú se incrementaron en 0.73\% respecto al año 2015.

\section{Figura 02}

Evolución de la pobreza y pobreza extrema en el Perú: 2009 - 2016 (\%).

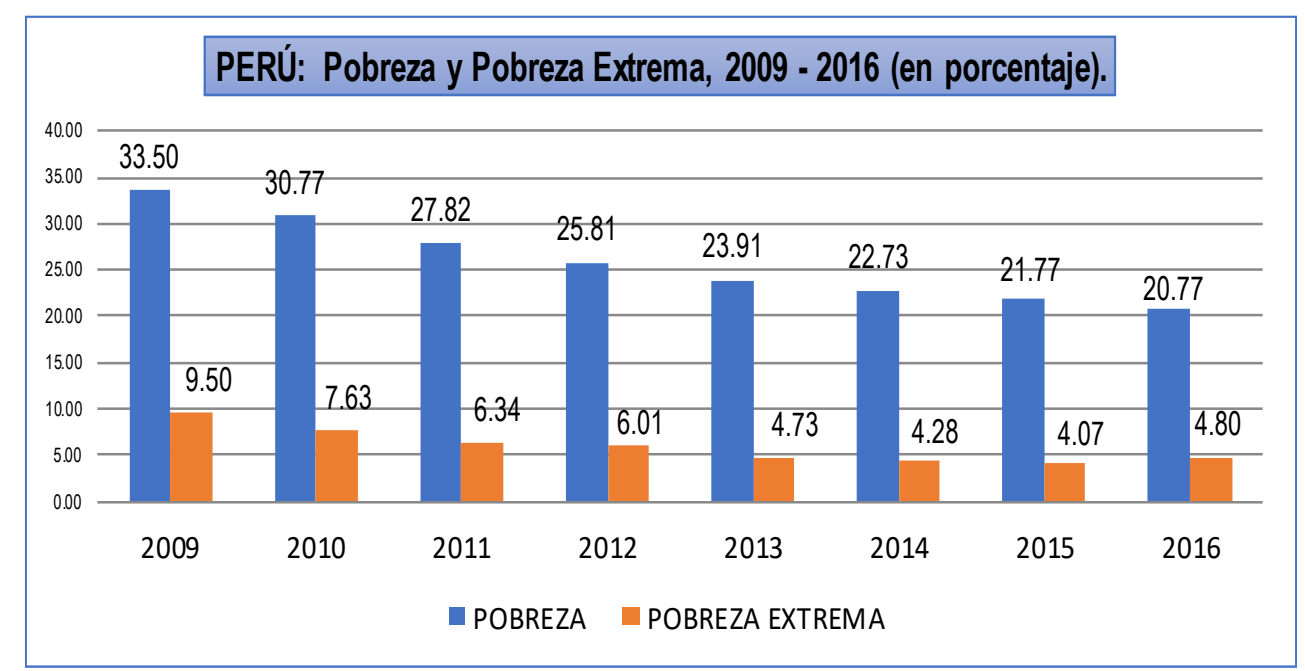

Fuente: INEI. Tomado de Plades (2016).

La evolución de la tasa de empleo en el Perú ha sido decreciente durante el periodo 2010 al 2015, observándose un incremento del 0.1\% en el año 2016; en cuanto a la brecha de empleo según género, se observa que decreció ligeramente en 17.7 puntos en el año 2016, es decir, en $0.4 \%$. Ver la Figura 03.

\section{Figura 03}

Evolución del empleo en el Perú: 2010 - 2016 (\%).

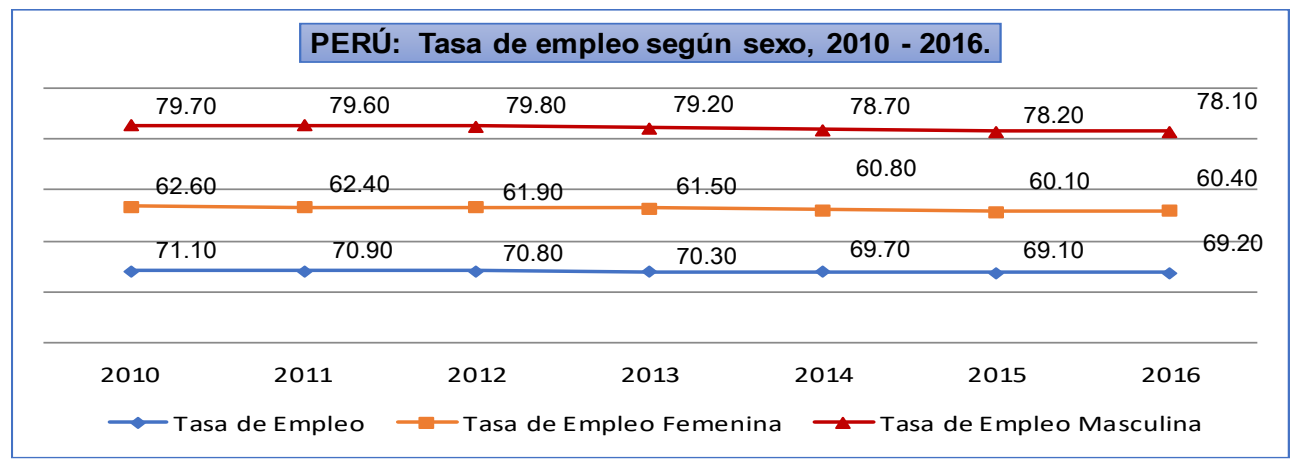

Fuente: INEI. Tomado de Plades (2016).

Por su parte, en el año 2016, la tasa de desempleo se incrementó en $0.7 \%$ revirtiendo la tendencia decreciente observada desde el año 2013 al año 2015; y en el caso de los hombres, el desempleo se incrementó en 1\%; mientras que el desempleo en las mujeres se incrementó en $0.5 \%$ respectivamente. Ver la Figura 04.

\section{Figura 04}

Evolución del desempleo en el Perú: 2010 - 2016 (\%). 


\begin{tabular}{|c|c|c|c|c|c|c|}
\hline \multicolumn{7}{|c|}{ PERÚ: Tasa de desempleo según sexo, 2010 - 2016.} \\
\hline 3.60 & 3.70 & 3.20 & 3.40 & 3.40 & \multicolumn{2}{|r|}{3.90} \\
\hline 4.70 & 4.40 & 4.40 & 4.70 & 4.00 & 3.60 & 4.60 \\
\hline 4.10 & 4.00 & 3.70 & 4.00 & 3.70 & 3.50 & 4.20 \\
\hline 2010 & 2011 & 2012 & 2013 & 2014 & 2015 & 2016 \\
\hline \multicolumn{7}{|c|}{$\multimap$ Tasa de Desempleo $\quad \longrightarrow$ Tasa de Desempleo en los hombres } \\
\hline \multicolumn{7}{|c|}{-Tasa de Desempleo en las mujeres } \\
\hline
\end{tabular}

Fuente: INEI. Tomado de Plades (2016).

En el año 2016, la población económicamente activa (PEA) ocupada eran 16,197 personas, asimismo, el $28.2 \%$ de estas personas laboraba en el segmento de servicios no personales, el $25.3 \%$ en los sectores de agricultura, ganadería y silvicultura; el $18.3 \%$ laboraba en el sector comercio, y el restante $28.2 \%$ laboraba en los segmentos minería, industria, construcción, servicios personales y los hogares, respectivamente. Ver la Figura 05.

\section{Figura 05}

Distribución de la PEA Ocupada según actividad en el Perú: 2010 - 2016 (\%).

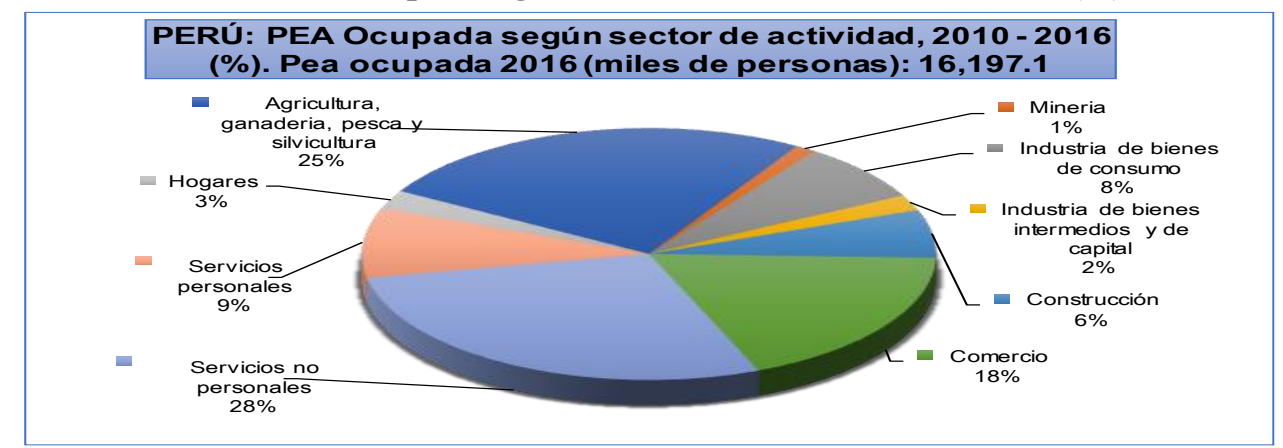

Fuente: INEI. Tomado de Plades (2016).

La informalidad laboral puede ser relacionada con diversos indicadores, uno de ellos es el trabajo no registrado. En el año 2016, la PEA asalariada con contrato laboral aumentó en $0.9 \%$ respecto al año anterior, esto confirma, que la PEA asalariada sin contrato ha disminuido porcentualmente en 5.3\% durante el periodo 2010 al 2016. Ver la Figura 06.

\section{Figura 06}

Población asalariada con contrato y sin contrato laboral en el Perú: 2010 - 2016 (\%).

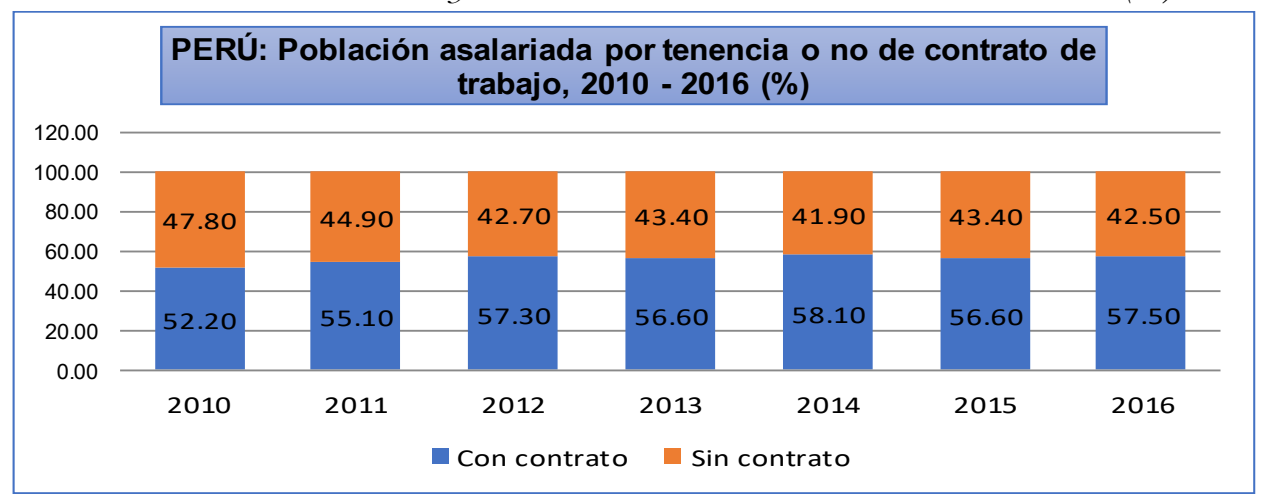

Fuente: INEI. Tomado de Plades (2016). 
De igual manera, durante el periodo 2010 al 2016, el empleo informal en el Perú alcanzó altos niveles, en este periodo, el empleo informal alcanzó tasas anuales superiores al $70 \%$, aunque durante estos últimos siete años, la tasa de empleo informal disminuyó en $5.1 \%$, respectivamente. Ver la Figura 07.

\section{Figura 07}

Población ocupada con empleo informal en el Perú: 2010 - 2016 (\%).

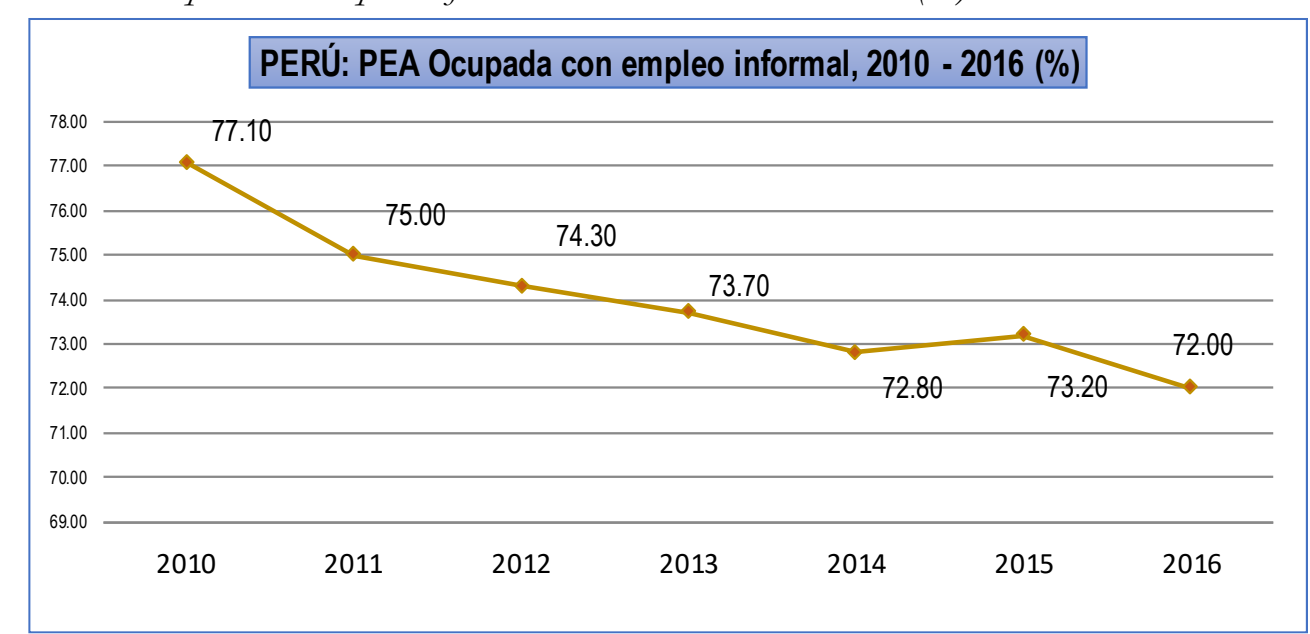

Fuente: INEI. Tomado de Plades (2016).

En tercer lugar, respecto a las remuneraciones en el Perú. Se observa que los ingresos monetarios de la PEA ocupada han tenido una tendencia creciente durante el periodo 2010 al 2016, estos ingresos han aumentado en S/166.00 a lo largo del periodo. Respecto a la brecha salarial por género, ésta se redujo de 36.3\% en el año 2010 a 29.2\% en el año 2016, sin embargo, estas estadísticas indican que la desigualdad de los ingresos entre hombres y mujeres en el Perú todavía siguen siendo significativas. Ver la Figura 08.

\section{Figura 08}

Ingreso real promedio de la Población ocupada en el Perú: 2010 - 2016 (S/).

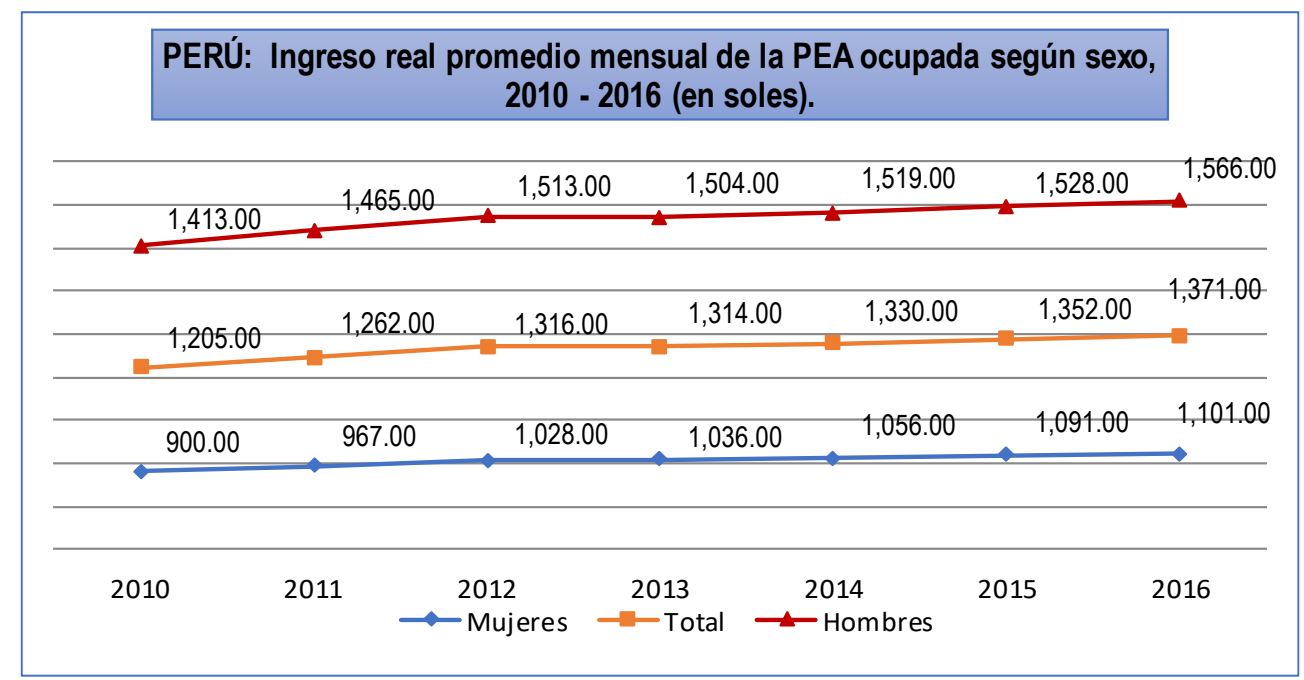

Fuente: INEI. Tomado de Plades (2016).

Asimismo, las actividades económicas con mayores ingresos monetarios en el Perú son la minería $(\mathrm{S} / 3,301.00)$ y la industria de bienes intermedios y de capital $(\mathrm{S} / 2,007.00)$, 
mientras que las actividades económicas que reportan menos ingresos a sus trabajadores son la agricultura, ganadería, silvicultura y la pesca con S/680.00. Ver la Figura 09.

\section{Figura 09}

Ingreso promedio por actividad económica en el Perú: 2016 (S/).

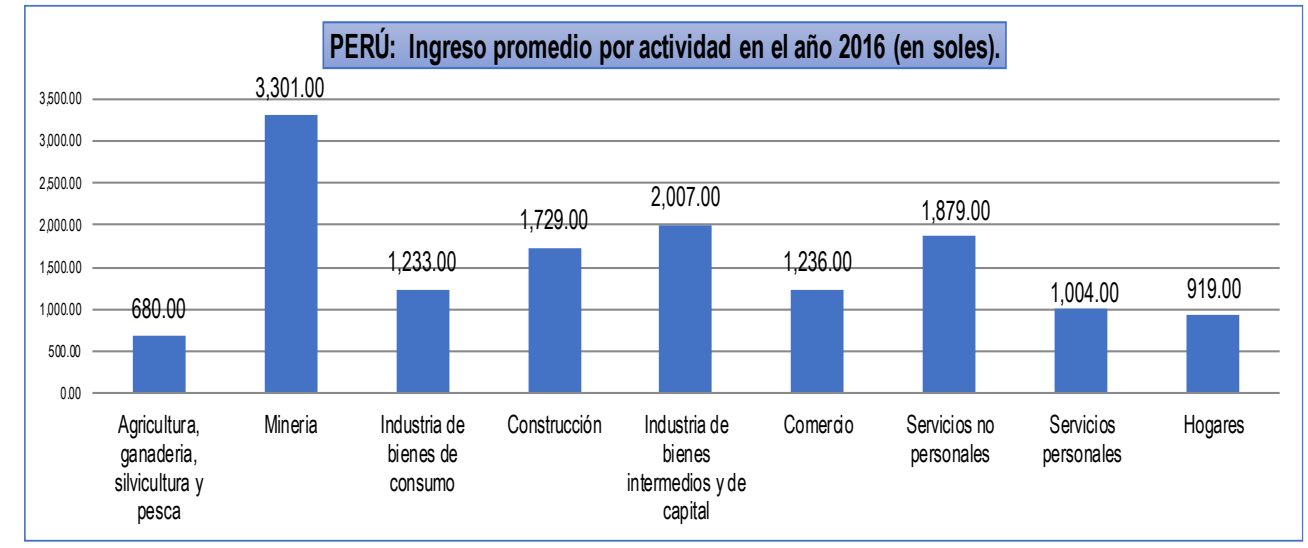

Fuente: INEI. Tomado de Plades (2016).

En cuarto lugar, respecto a la seguridad social en el Perú. Existen dos sistemas de pensiones en el Perú: el Sistema Nacional del Pensiones (SNP) y el Sistema Privado de Pensiones (SPP). En este sentido, sólo el 34.5\% del total de la PEA ocupada aporta a un sistema de pensiones, cifra superior en $4.9 \%$ respecto a la cifra registrada en el año 2010. Ver la Figura 1

\section{Figura 10}

Población ocupada que aporta a algún sistema de pensiones en el Perú: 2010 - 2016 (S/).

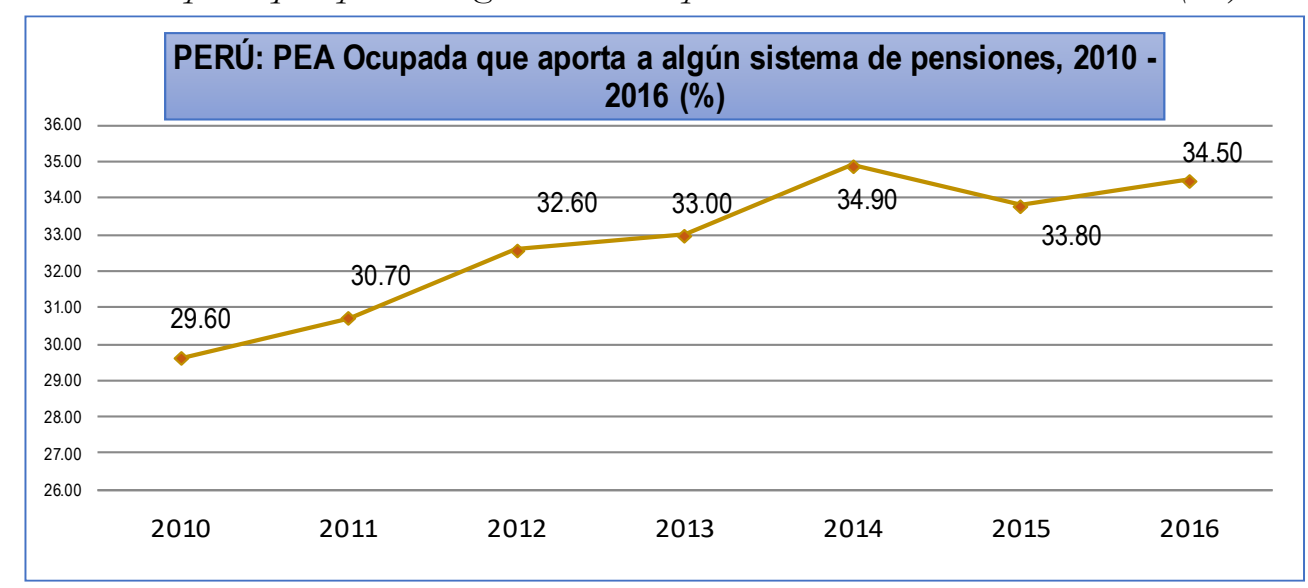

Fuente: INEI. Tomado de Plades (2016).

En cuanto a la tasa de sindicalización de los trabajadores en el Perú, se observa una tendencia decreciente en el año 2016 en 0.3 puntos porcentuales respecto al año anterior. De igual manera, en el Sector Privado se observa una fuerte caída $0.4 \%$ en la tasa de sindicalización en ese año. Ver la Figura 11.

\section{Figura 11}

Tasa de sindicalización de la PEA asalariada en el Perú: 2014 - 2016 (S/). 


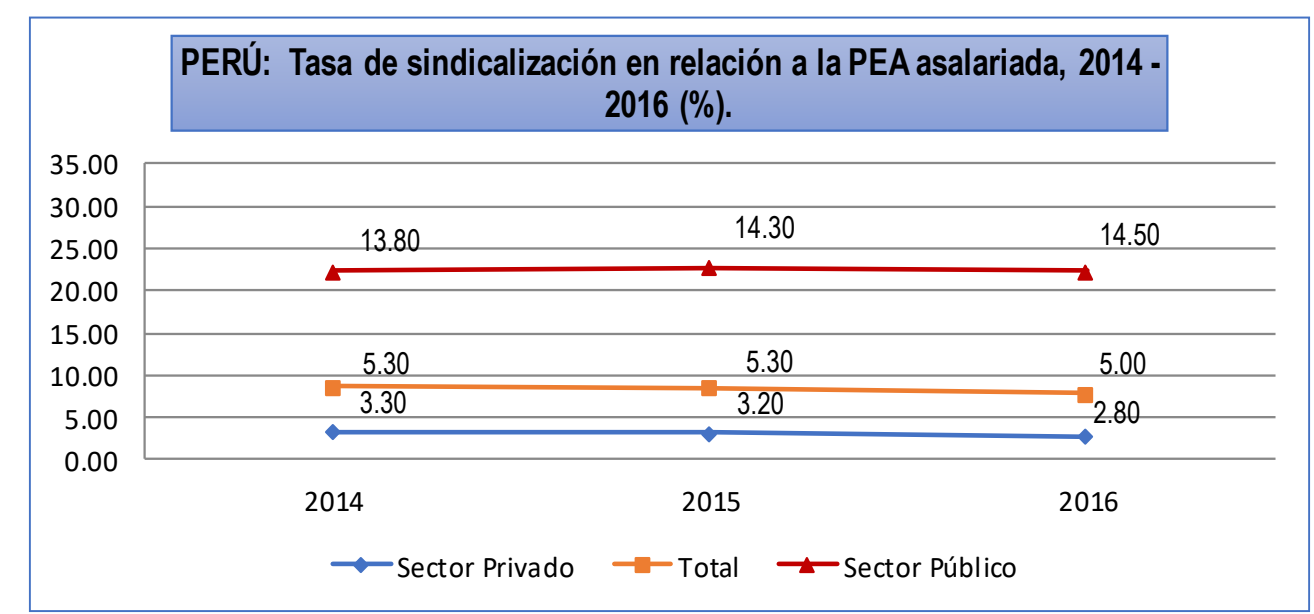

Fuente: MTPE. Tomado de Plades (2016).

En quinto lugar, respecto a la situación de los Pliegos de Reclamos Laborales y Negociaciones Colectivas en el Perú. En el año 2016, se presentaron 548 pliegos de reclamos, cifra menor en comparación al año anterior, asimismo, se registraron 352 convenios colectivos en este mismo año, es decir, se observó un mínimo incremento de 20 convenios respecto al año 2015, estas cifras reflejan el impacto que han tenido las políticas laborales desde el año 2012.

\section{Figura 12}

Número de Pliegos de Reclamos presentados y Convenios Colectivos registrados en el Perú: 2010 - 2016.

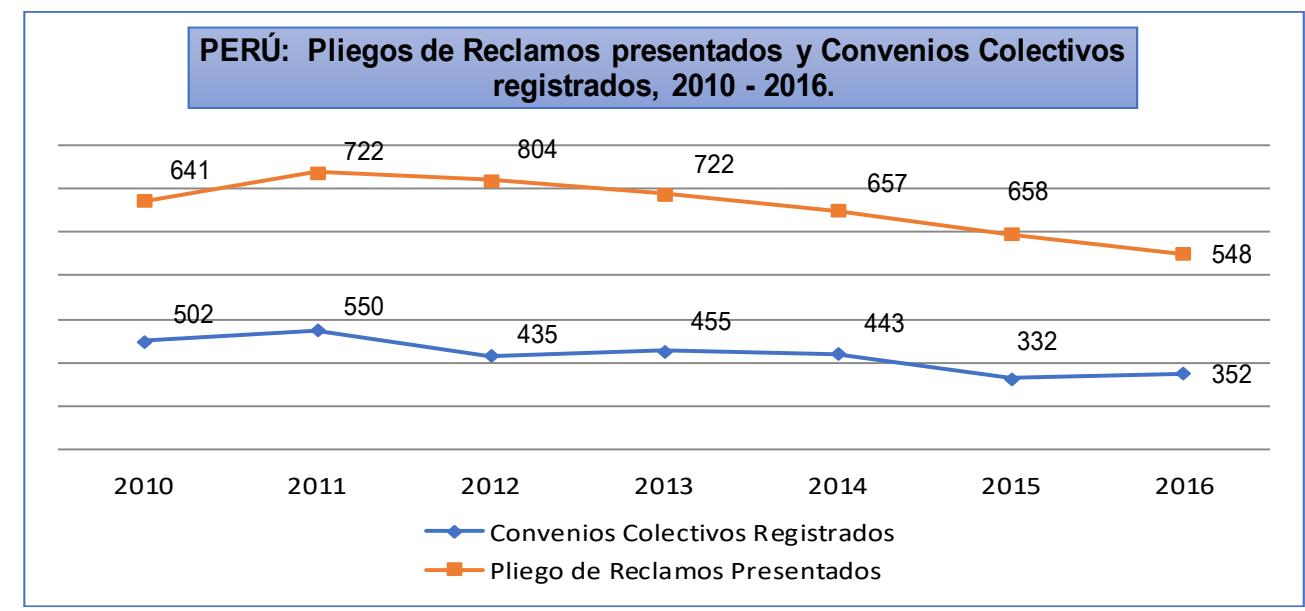

Fuente: MTPE. Tomado de Plades (2016).

En síntesis, según la revisión de algunos indicadores socioeconómicos del país, nos indican que el panorama observado del trabajo decente en el Perú resulta ser poco auspicioso en el presente, pese al crecimiento de la economía peruana en el año 2016, este nivel de crecimiento se encuentra muy alejado de los niveles logrados en los años 2008 y 2010, este débil crecimiento tuvo un impacto moderado en la pobreza disminuyéndola en $1 \%$ y ningún impacto positivo en la lucha contra la pobreza extrema. Los niveles de empleo en el país han mejorado únicamente en $0.1 \%$ y la brecha de empleo según genero decreció en $0.4 \%$, sin embargo, la tasa de desempleo en el país se incrementó en $0.7 \%$, de igual manera, la informalidad en el Perú continua siendo un grave problema en nuestra economía, puesto que durante el periodo 2010 al 2016, más del 40\% de la población 
asalariada en el país trabaja sin ningún tipo de contrato laboral y más del 70\% de la PEA ocupada tiene empleo informal.

Por otro lado, en sectores importantes de nuestra economía como la agricultura, la ganadería y la pesca, los trabajadores solamente reciben un ingreso promedio de S/680.00 mensuales -ingreso monetario que no les permitiría cubrir sus necesidades básicas-. Además, las estadísticas de la seguridad social en el Perú indican que únicamente el 34.50\% de la PEA ocupada aporta para sus pensiones de jubilación. Finalmente, los niveles de sindicalización laboral en el país han disminuido principalmente en el sector privado (menos $0.4 \%$ en el año 2016), de igual manera, el número de pliegos de reclamos laborales presentados y los convenios colectivos registrados en el país presentan una tendencia decreciente durante el periodo del año 2010 al año 2016.

\section{TENDENCIAS LABORALES FUTURAS Y EL TRABAJO DECENTE}

Términos como robotización, digitalización o inteligencia artificial se están popularizando en nuestro vocabulario y son usados para describir los cambios vertiginosos que se están produciendo en el mundo del trabajo y en las economías en el siglo XXI, uno de estos cambios se puede percibir en las formas atípicas de empleo que vienen surgiendo pero que tienden a debilitar los derechos laborales tradicionales debido a la fragmentación de los procesos productivos en un contexto digital y globalizado que están modificando la organización de la producción y el trabajo que escapan al marco legal tradicional de relaciones laborales, en este contexto cambiante, el reto hacia adelante, es hacer efectivo el horizonte del trabajo decente para el bienestar de nuestras sociedades. Las revoluciones tecnológicas de los siglos pasados han provocado cambios en el empleo, e inclusive han creado más empleo, sin embargo, las innovaciones tecnológicas del presente, vienen provocando cambios e impactos a mayor velocidad nunca antes vistos en el empleo por efecto simultaneo de la globalización y la digitalización.

Si se echa una mirada atrás, es incuestionable que han producido avances sociales, sin embargo, todavía persisten los déficits de trabajo decente a nivel mundial entendiendo que el trabajo decente es el trabajo con derechos, sin discriminación, en condiciones saludables, con una remuneración que permita vivir dignamente y con protección social, el panorama se aclara si se revisan algunas estadísticas: cerca de 152 millones de niños y niñas se encuentran atrapados en el trabajo infantil, 25 millones de personas tienen trabajos forzosos, el $60 \%$ de los 3.300 millones de trabajadores potenciales no tienen trabajo o tienen un trabajo informal, actualmente hay más de 245 millones de migrantes que son más del $41 \%$ en comparación al siglo pasado, 800 millones de personas aun contando con un trabajo se encuentran en situación de pobreza extrema. Los objetivos de desarrollo sostenible marcan que los próximos años se debe erradicar la pobreza extrema y reducir a la mitad la pobreza relativa, para ello, se debe mejorar los salarios: incrementar los salarios mínimos y mejorar los salarios reales (Nieto, 2017)

\section{CONCLUSIONES}

El trabajo es un elemento fundamental en la vida cotidiana de las personas, es por eso que es necesario que el trabajo se caracterice por ser digno, justo, apropiado, de calidad y de cantidad suficiente, de tal manera, que el trabajador pueda satisfacer sus necesidades 
y las de su familia de la mejor manera posible, esta es la noción del concepto de trabajo decente, esta concepción permite diferenciar los tipos de trabajo que no ofrecen condiciones mínimas de calidad para los trabajadores versus los trabajos que sí permiten desarrollar las capacidades de las personas, elevar su productividad y que les permita ganarse la vida satisfactoriamente, es por eso que, el trabajo decente representa un camino hacia una sociedad más equitativa, por tanto, el trabajo decente encarna un anhelo para todo aquel país que busque verdaderamente el desarrollo y el bienestar de su población como miras al futuro. Sin embargo, la problemática del mundo actual como la pobreza, la discriminación, la desigualdad de ingresos, la sobrexplotación, la informalidad, etc. afecta la realización del trabajo decente, es por ello que se requiere de un marco adecuado de políticas nacionales e internacionales que dejen de concebir el trabajo como una simple mercancía o un elemento de un mercado de bienes o servicios.

En el caso del Perú, se ha determinado que existe un déficit de trabajo decente que es muy significativo y preocupante en relación al futuro de nuestra sociedad, principalmente a causa de los altos niveles de informalidad presentes en nuestra economía que han llegado a superar el 70 por ciento de la población ocupada en el año 2016 y a los altos niveles registrados de pobreza que alcanza al 20.77 por ciento de la población peruana. Asimismo, existe una baja cobertura de protección social en el país, puesto que solamente el 34.50 por ciento de la población ocupada aporta a un sistema de pensiones; los trabajadores de algunos sectores importantes de la economía como la agricultura, ganadería y la pesca reciben un ingreso promedio de S/680.00 mensual muy por debajo de la remuneración mínima legal; en cuanto a los índices de dialogo social como las tasas de sindicalización laboral y los convenios colectivos a nivel nacional vienen presentando una tendencia decreciente desde el año 2010. Por su parte, Gamero (2014) determinó que, en el año 2013, solamente el $13 \%$ de la población ocupada en el Perú cuenta con un empleo que cumple con las condiciones básicas de un trabajo decente con contrato de trabajo, ingresos mayores que el salario mínimo, una jornada laboral de 48 horas semanales, con seguro de salud y aporta a algún sistema de pensiones. En cuanto al índice de trabajo decente a nivel regional en el Perú, Goyzueta et al (2016) estimaron que la región con mayor IDT es Lima con 0.6609 puntos versus la región con menor IDT que es Huancavelica con 0.5076 puntos, según lo cual se evidencia que las buenas condiciones laborales están relacionadas con los niveles de competitividad regional, por lo cual, las políticas públicas deben orientarse a mejorar los componentes del trabajo decente como el empleo y la educación.

Los avances tecnológicos del siglo XXI vienen provocando una serie de cambios en diversos aspectos de la vida de las personas y las sociedades, los campos del trabajo y del empleo no son la excepción, la aparición de formas atípicas de trabajo como la gigeconomy, el crowdworking y la uberización que se han extendido por todo el mundo tienden a debilitar los derechos laborales tradicionales o difieren de la relación laboral estándar en la que se contaba con un contrato indefinido o una determinada jornada laboral, estos empleos atípicos se caracterizan por ser trabajos estacionales, ocasionales, se realizan a través de una intermediación laboral o se manejan a través de una plataforma o un aplicativo informático; todos estos cambios significan afrontar una serie de desafíos que garanticen: los derechos laborales, la igualdad de trato para todos los trabajadores, las 
negociaciones colectivas, el acceso a la protección social y a la salud, entre otros, para ello, se necesita de la formulación de un nuevo tipo políticas públicas basadas en la declaración universal de los derechos humanos y pactos internacionales, este tipo de políticas deben de reconocer el rol que el trabajo decente tiene en la estabilidad social, la paz, la productividad y la competitividad que son factores fundamentales en el crecimiento sostenido de las sociedades.

\section{REFERENCIAS BIBLIOGRÁFICAS}

Ballestero Bascuas, C. (2016). El trabajo decente: ¿la aspiración del siglo XXI? Segovia, España: Universidad de Valladolid.

Egger, P., \& Sengenberger, W. (2002). Problemas y políticas del trabajo decente. Boletín Cinterfor(153), pp. 27-67.

Ermida Uriarte, O. (2001). Trabajo decente y formación profesional. CINTERFOR, Boletín interno(151),pp. 9-26.

Gálvez Santillán, E., Gutiérrez Garza, E., \& Picazzo, P. E. (2011). El trabajo decente: nuevo paradigma para el fortalecimiento de los derechos sociales. Revista Mexicana de Sociología, 73(1), pp. 73-104.

Gálvez Santillán, E., Gutiérrez Garza, E., Picazzo Palencia, E., \& Osorio Calderón, J. (2016). El trabajo decente, una alternativa para reducir la desigualdad en la globalizacion: el caso de México. Revista Región y Sociedad, XXV III(66), pp. 55-94.

Gamero Requena, J. (2014). El Trabajo Decente en el Perú: una mirada al 2013.

Ghai, D. (2003). Trabajo decente. Conceptos e indicadores. Revista Internacional del Trabajo, 122(2), pp. 125-160.

Goyzueta Torres, G., Tudela Mamani, J., \& Canahuire Sejje, E. (2016). Índice de Trabajo Decente en el Perú: Elaboración y Analisis Comparativo a Nivel Regional. Revista de Investigación Altoandina, 18(1), pp. 37-46.

Lanari, M. (2005). Trabajo decente: significados y alcances del concepto. Indicadores propuestos para su medición. Buenos Aires, Agentina: Ministeiro de Trabajo, Empleo, y Seguridad Social.

Nieto, J. (2017). Tendencias laborales y el futuro del trabajo. Gaceta Sindical(29), pp. 3142.

OIT. (31 de mayo de 2017). El trabajo decente crucial para el desarrollo sostenible. Obtenido de https://www.ilo.org/global/topics/sdg-2030/resources/WCMS_470340/lang-es/index.htm

OIT. (11 de Noviembre de 2018). Trabajo decente y la Agenda 2030 de desarrollo sostenible. Obtenido de https://www.ilo.org/global/topics/sdg-2030/lang--es/index.htm

Plades. (2016). Informe sobre situación del trabajo decente en el Perú 2016. Programa Laboral de Desarrollo.

Rodgers, G. (2002). El trabajo decente como una meta para la economia global. Boletin Cinterfor, 153, 9-28. 
Somavía, J. (2001). Reducir el déficit de trabajo decente: un desafio global. Boletín Cinterfor(151), 177-186.

\section{CITAR COMO:}

Quispe Quezada, U. R., \& Villarroel Díaz, V. R. (2019). El panorama del trabajo decente en el Perú durante el periodo 2010 al 2016. Puriq, 1(02), 133-146. https://doi.org/10.37073/puriq.1.02.47 